\title{
Dehn surgery on knots of wrapping number 2
}

\author{
YING-QING WU
}

\begin{abstract}
Suppose $K$ is a hyperbolic knot in a solid torus $V$ intersecting a meridian disk $D$ twice. We will show that if $K$ is not the Whitehead knot and the frontier of a regular neighborhood of $K \cup D$ is incompressible in the knot exterior, then $K$ admits at most one exceptional surgery, which must be toroidal. Embedding $V$ in $S^{3}$ gives infinitely many knots $K_{n}$ with a slope $r_{n}$ corresponding to a slope $r$ of $K$ in $V$. If $r$ surgery on $K$ in $V$ is toroidal then either $K_{n}\left(r_{n}\right)$ are toroidal for all but at most three $n$, or they are all atoroidal and nonhyperbolic. These will be used to classify exceptional surgeries on wrapped Montesinos knots in a solid torus, obtained by connecting the top endpoints of a Montesinos tangle to the bottom endpoints by two arcs wrapping around the solid torus.
\end{abstract}

$57 \mathrm{~N} 10$

\section{Introduction}

A Dehn surgery on a hyperbolic knot $K$ in a compact 3-manifold is exceptional if the surgered manifold is nonhyperbolic. When the manifold is a solid torus, the surgery is exceptional if and only if the surgered manifold is either a solid torus, reducible, toroidal, or a small Seifert fibered manifold whose orbifold is a disk with two cone points. Solid torus surgeries have been classified by Berge [1], Gabai [7; 8] and Scharlemann [19]; there is no reducible surgery. For toroidal surgery, Gordon and Luecke [11] showed that the surgery slope must be either an integral or a half integral slope. By Gordon and the author [12], this is also true for small Seifert fibered surgeries.

In this paper we study Dehn surgery on hyperbolic knots $K$ in a solid torus $V$ with wrapping number 2 . The wrapping number $\operatorname{wrap}(K)$ of a knot $K$ in a solid torus $V$ is defined to be the minimal geometric intersection number of $K$ with a meridional disk $D$ of $V$, and the winding number wind $(K)$ of $K$ is the algebraic intersection number of $K$ with $D$. Thus if $K$ is a knot in a solid torus $V$ with $\operatorname{wrap}(K)=2$ then $\operatorname{wind}(K)=0$ or 2 . It follows from the results above that there is no reducible or solid torus surgery on such a hyperbolic knot. We would like to know if there are toroidal or small Seifert fibered surgeries on such a knot. 
Exceptional surgery does exist on some knots with wrapping number 2 . If $K$ in $V$ has a spanning surface which is either a punctured torus or a punctured Klein bottle then surgery on $K$ along the boundary slope of this surface is toroidal. A well-known example of knots in solid tori that admit multiple exceptional surgeries is the Whitehead knot, obtained by deleting an open neighborhood of a component of the Whitehead link in $S^{3}$. It admits a total of five exceptional surgeries, two toroidal and three small Seifert fibered.

For the case of knots with winding number 2, consider the knot obtained by putting a Montesinos tangle $T[-1 / 2,1 / 3]$ horizontally in the solid torus $V$ and then connecting the top endpoints to the bottom endpoints by two strings running around the solid torus; see Figure 5.1(b), where $V$ is the complement of the dotted circle. It is called a wrapped Montesinos knot and denoted by $K^{1}(-1 / 2,1 / 3)$; see Section 5 for more details. We will show that this knot admits three exceptional surgeries, two toroidal and one small Seifert fibered; see Proposition 2.2. We suspect that these are the only examples of knots with wrapping number 2 that admit multiple exceptional surgeries.

Conjecture 1.1 Suppose that $K$ is a hyperbolic knot of wrapping number 2 in a solid torus $V$ and $K$ is not the Whitehead knot or the wrapped Montesinos knot $K^{1}(-1 / 2,1 / 3)$. Then $K$ admits no small Seifert fibered surgery and at most one toroidal surgery.

Let $D$ be a meridian disk intersecting $K$ twice. Cutting $(V, K)$ along $D$ produces a 2 -string tangle $(B, \tau)$. Let $X$ be the tangle space $B-\operatorname{Int} N(\tau)$, and let $\partial_{h} X$ be the frontier of $X$ in $V$. It can be shown that for the knot $K=K^{1}(1 / 2,1 / 3)$ above, this surface $\partial_{h} X$ is compressible. This is a very special property since most 2-string tangle spaces have incompressible boundary. For example, if $\tau$ is a Montesinos tangle of length at least 2 then $\partial(B-\operatorname{Int} N(\tau))$ is incompressible unless $\tau$ is equivalent to $T[1 / 2, p / q]$; see the author [23]. The following theorem shows that the above conjecture is true if $\partial_{h} X$ is incompressible. Denote by $(V, K, r)$ the manifold obtained by $r$ surgery on a knot $K$ in a 3 -manifold $V$.

Theorem 3.8 Suppose $K$ is a hyperbolic knot with $\operatorname{wrap}(K)=2$ in a solid torus $V, K$ is not the Whitehead knot, and $\partial_{h} X$ is incompressible in $X$. Then $K$ admits at most one exceptional surgery $(V, K, r)$, which must be a toroidal surgery and $r$ an integral slope.

Note the surface $\partial_{h} X$ is always incompressible if $K$ is hyperbolic and $\operatorname{wind}(K)=0$, hence the above theorem shows that Conjecture 1.1 is true for knots with $\operatorname{wind}(K)=0$. Since the Whitehead knot admits five exceptional surgeries, it is surprising to see that 
no other knots with wind $(K)=0$ and $\operatorname{wrap}(K)=2$ have more than one exceptional surgeries.

We now consider knots obtained by embedding $(V, K)$ in the 3 -sphere. Let $\varphi_{0}$ be a standard embedding, and $\varphi_{n}$ the composition of $\varphi_{0}$ with $n$ right hand full twists of $V$ along a meridian disk. Denote by $K_{n}=\varphi_{n}(K)$ and by $r_{n}=\varphi_{n}(r)$, for a fixed slope $r$ of $K$. Denote by $K_{n}\left(r_{n}\right)$ the surgery on $K_{n}$ along the slope $r_{n}$. Clearly $K_{n}\left(r_{n}\right)$ is obtained by Dehn filling $(V, K, r)$ on $\partial V$, hence if $(V, K, r)$ is hyperbolic then most $K_{n}\left(r_{n}\right)$ are hyperbolic. In general it might be possible that $(V, K, r)$ is nonhyperbolic while infinitely many $K_{n}\left(r_{n}\right)$ are hyperbolic. However, we will show that this does not happen when $\operatorname{wrap}(K)=2$.

Theorem 4.3 Suppose $\operatorname{wrap}(K)=2$, and $(V, K, r)$ is nonhyperbolic. Then $K_{n}\left(r_{n}\right)$ is nonhyperbolic for all but at most three $n$. Moreover, one of the following holds:

(1) there is an $n_{0}$ such that $K_{n}\left(r_{n}\right)$ is toroidal unless $\left|n-n_{0}\right| \leq 1$;

(2) $K_{n}\left(r_{n}\right)$ is atoroidal for all $n$, and there exist $q_{1}, q_{2} \in \mathbb{Z}$ such each $K_{n}\left(r_{n}\right)$ is either reducible or has a small Seifert fibration with $q_{1}, q_{2}$ as the indices of two of its singular fibers.

Thus if $(V, K, r)$ is nonhyperbolic then $K_{n}\left(r_{n}\right)$ is either toroidal for all but at most three $n$, or is never toroidal. This property is useful in determining whether $(V, K, r)$ is hyperbolic; see the proof of Theorem 5.5.

Up to homeomorphism there are essentially two ways to make wrapped Montesinos links from a Montesinos tangle $T\left[t_{1}, \ldots, t_{k}\right]$, denoted by $K^{0}\left[t_{1}, \ldots, t_{k}\right]$ and $K^{1}\left[t_{1}, \ldots, t_{k}\right]$; see Section 5 for detailed definitions. The above theorems will be used to prove the following classification theorem, which shows that there are no other exceptional Dehn surgeries on wrapped Montesinos knots in solid tori besides the well-known examples and the ones mentioned above. In particular, Conjecture 1.1 is true for these knots. Here two pairs $(K, r)$ and $\left(K^{\prime}, r^{\prime}\right)$ are equivalent if there is an obvious homeomorphism of $V$ taking one to the other; see Section 5 for detailed definitions. We may assume that $K \neq K^{a}[0]$ or $K^{a}[1 / q]$ as otherwise $K$ is nonhyperbolic.

Theorem 5.5 Suppose $K=K^{a}\left(t_{1}, \ldots, t_{k}\right) \subset V$ is not equivalent to $K^{a}(0)$ or $K^{a}(1 / q)$ for any integer $q$. Let $(V, K, r)$ be an exceptional surgery. Then $(K, r)$ is equivalent to one of the following pairs. The surgery is small Seifert fibered for $r=1,2,3$ in (1) and $r=7$ in (4), and toroidal otherwise.

(1) $K=K^{0}(2)$ (the Whitehead knot), $r=0,1,2,3,4$.

(2) $K=K^{a}(n), n>2, r=0$ if $a=0$, and $r=2 n$ otherwise.

(3) $K=K^{a}\left(1 / q_{1}, 1 / q_{2}\right),\left|q_{i}\right| \geq 2$, and $r$ is the pretzel slope.

(4) $K=K^{1}(-1 / 2,1 / 3), r=6,7,8$. 
These results will be used to study Seifert fibered surgery on Montesinos knots in $S^{3}$. We will show that $6+4 n$ and $7+4 n$ surgeries on hyperbolic $(-2,3,2 n+1)$ pretzel knots are Seifert fibered; see Corollary 2.3 below. It will be proved in a forthcoming paper by the author [21] that there are only finitely many other Seifert fibered surgeries on hyperbolic Montesinos knots of length 3.

\section{Preliminaries and examples}

Given a submanifold $F$ of a manifold $M$, let $N(F)$ be a regular neighborhood of $F$ in $M$. When $F$ has codimension 1 and is properly embedded, denote by $M \mid F$ the manifold obtained by cutting $M$ along $F$. If $K$ is a knot in $M$, denote by $(M, K, r)$ the manifold obtained from $M$ by Dehn surgery on $K$ along a slope $r$ on $\partial N(K)$. When $M=S^{3}$, simply denote $\left(S^{3}, K, r\right)$ by $K(r)$.

A cusped manifold is a compact 3-manifold $M$ with a specified vertical boundary $\partial_{v} M$, which is a disjoint union of annuli and tori on $\partial M$. The closure of $\partial M-\partial_{v} M$ is the horizontal boundary of $M$, denoted by $\partial_{h} M$. If $M$ is an $I$-bundle over a compact surface $F$ then it has a natural cusped manifold structure with $\partial_{v} M$ the annuli over $\partial F$. Conversely, a cusped manifold $M$ is considered an $I$-bundle only if it is an $I$-bundle with $\partial_{v} M$ defined above. A surface $F$ properly embedded in $M$ with $\partial F \subset \partial_{h} M$ is an $h$-essential surface if it is incompressible, and has no boundary compressing disk disjoint from $\partial_{v} M$.

Let $K$ be a hyperbolic knot in a solid torus $V$ with $\operatorname{wrap}(K)=2$. Let $D$ be a meridional disk of $V$ intersecting $K$ twice. Cutting $V$ along $D$, we obtain a 3-ball $B$. Let $\tau=K \cap B$ be the 2 -string tangle in $B$. Denote by $X=B-\operatorname{Int} N(\tau)$ the tangle space. Clearly $X$ is irreducible, and the hypothesis that $K$ is hyperbolic implies that $X$ is also atoroidal. Define the vertical boundary of $X$ to be $\partial_{v} X=\partial V \cap X$. Then the horizontal boundary $\partial_{h} X$ is the disjoint union of two copies of a once punctured torus when $\operatorname{wind}(K)=0$, or a single twice punctured torus when $\operatorname{wind}(K)=2$.

Let $Y=N(D \cup K)$ and define $\partial_{v} Y=\partial V \cap Y$. Then we can write $V=X \cup_{\eta} Y$, where $\eta: \partial_{h} X \rightarrow \partial_{h} Y$ is a homeomorphism. The surgery manifold can then be written as $(V, K, r)=X \cup_{\eta}(Y, K, r)$.

Fix a trivial embedding of $V$ in $S^{3}$. Let $K^{\prime}$ be the core of $S^{3}-V$. If $K$ is a knot in $V$ then $L=K^{\prime} \cup K$ is a link in $S^{3}$. Conversely, if $L=K^{\prime} \cup K$ is a two component link in $S^{3}$ and $K^{\prime}$ is trivial then $K$ is a knot in $V=S^{3}-\operatorname{Int} N\left(K^{\prime}\right)$. We use the convention that a trivial circle $K^{\prime}$ with a dot represents the component that need to be deleted, so the link $L=K^{\prime} \cup K \subset S^{3}$ represents the pair $(V, K)$ with $V=S^{3}-\operatorname{Int} N\left(K^{\prime}\right)$. 
The preferred meridian-longitude pair $(m, l)$ of $K$ in $S^{3}$ (see Rolfsen [18]) is then considered the preferred meridian-longitude pair of $K$ in $V$. This sets up a coordinate system for the slopes on $\partial N(K)$, so a slope $q l+p m$ is represented by a rational number $p / q$, or $1 / 0$ if $(p, q)=(1,0)$.

Let $C$ be the core of $V$. Fix a meridian-longitude pair $\left(m_{0}, l_{0}\right)$ of $\partial V$. We can re-embed $V$ in $S^{3}$ by an orientation preserving homeomorphism $\varphi_{n}: V \rightarrow V$ such that $l_{0}$ is mapped to the curve $l_{0}+n m_{0}$ on $\partial V$. Denote by $K_{n}=\varphi_{n}(K)$. Thus $K_{n}$ is obtained from $K$ by $n$ right hand full twists along a disk bounded by $K^{\prime}$. If $r$ is a slope on $\partial N(K)$, let $r_{n}$ be the corresponding slope $\varphi_{n}(r)$ on $\partial N\left(K_{n}\right)$. We have $r_{n}=r+n \times \operatorname{wind}(K)^{2}$, hence $r_{n}=r$ if $\operatorname{wind}(K)=0$, and $r_{n}=r+4 n$ if $\operatorname{wind}(K)=2$.

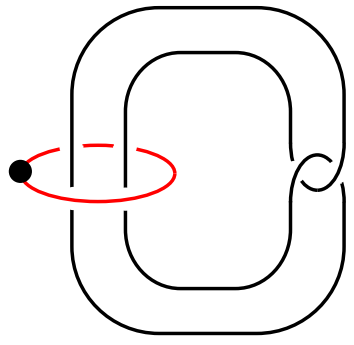

(a)

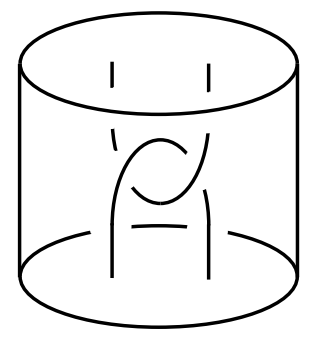

(b)

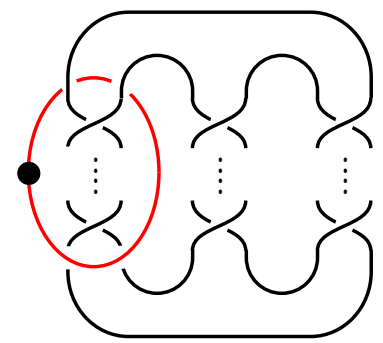

(c)

Figure 2.1

Example 2.1 (1) Let $K$ be the Whitehead knot in $V$ as shown in Figure 2.1(a). Then $(V, K, r)$ is toroidal for $r=0,4$, and is Seifert fibered for $r=1,2,3$; see Gordon and the author [13, Lemma 7.1] and Brittenham and the author [3, Lemma 2.3]. Cutting $(V, K)$ along a meridional disk produces a tangle $(B, \tau)$ as shown in Figure 2.1(b), which will be called the Whitehead tangle.

(2) Suppose $K$ has a spanning surface $F$ in $V$ which is a once punctured torus or Klein bottle with boundary slope $r$. Then $F$ becomes a closed surface $\widehat{F}$ in $(V, K, r)$, which is either a Klein bottle or a nonseparating torus. Since $(V, K, r)$ is irreducible [19], the boundary of a regular neighborhood of $\widehat{F}$ is incompressible in $(V, K, r)$, hence $(V, K, r)$ is toroidal. In particular, any hyperbolic pretzel knot in solid torus as shown in Figure 2.1(c) admits a toroidal surgery along the boundary of its pretzel surface.

Note that $K_{n}\left(r_{n}\right)$ is obtained from $(V, K, r)$ by attaching a solid torus to $\partial V$ along the slope $l_{0}-n m_{0}$ on $\partial V$, hence if $(V, K, r)$ is hyperbolic then $K_{n}\left(r_{n}\right)$ is hyperbolic for all but finitely many $n$. 


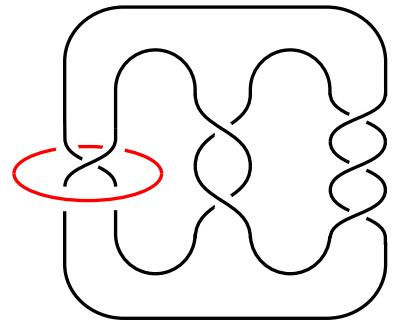

(a)

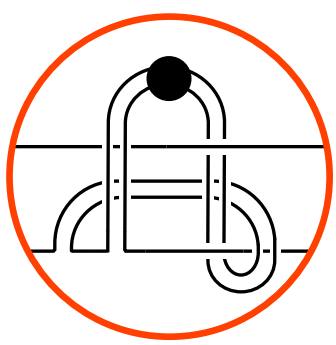

(d)

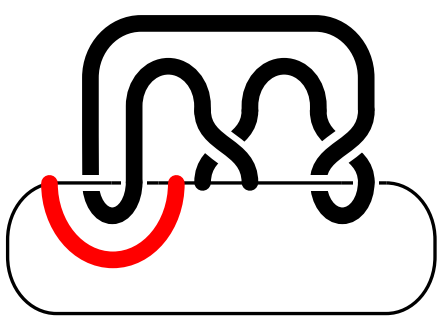

(b)

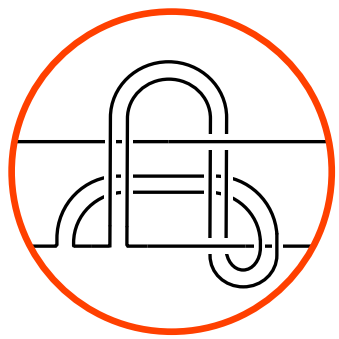

(e)

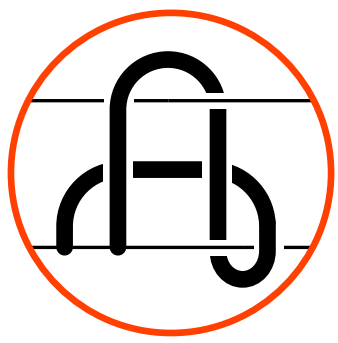

(c)

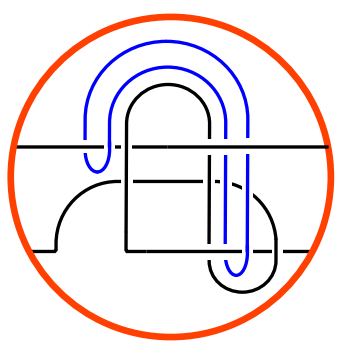

(f)

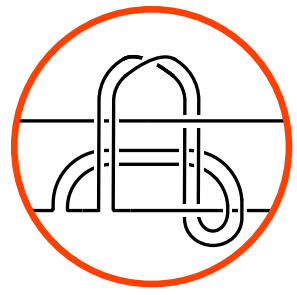

(g)

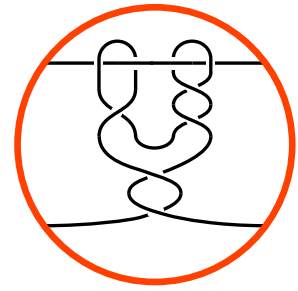

(h)

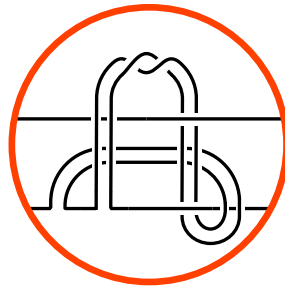

(i)

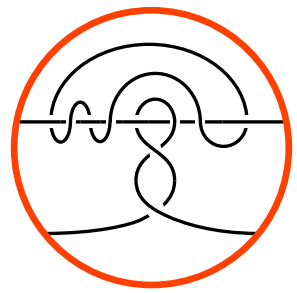

(j)

Figure 2.2

Proposition 2.2 Let $K$ be the knot in solid torus as shown in Figure 2.2(a). Then $(K, V, 8)$ and $(K, V, 6)$ are toroidal, and $(K, V, 7)$ is small Seifert fibered with two singular fibers of indices 3 and 5, respectively.

Proof Rotating along a horizontal axis of the knot diagram gives a double branched cover of $\left(S^{3}, L\right)$ over the pair $\left(S^{3}, \lambda\right)$ in Figure 2.2(b), where $\lambda$ is a pair of arcs represented by the thick curves in the figure. The thin circle $C$ in Figure 2.2(b) is the image of the axis and hence forms the branch set. The neighborhoods of the two components of $L=K^{\prime} \cup K$ in Figure 2.2(a) project to regular neighborhoods of $\lambda$, which are 3-balls $B_{1}, B_{2}$ respectively, where $B_{1}$ is represented by the lower thick arc 
in Figure 2.2(b). Let $B$ be the closure of the complement of $B_{1}$, and let $\tau=C \cap B$. Then $\left(B, B_{2}, \tau\right)$ can be deformed to that in Figure 2.2(c) and then further to that in Figure 2.2(d).

Since $V$ is the exterior of $K^{\prime}$, the above shows that $(V, N(K))$ is the double branched cover of $\left(B, B_{2}\right)$ branched over $\tau$. Put $\tau_{2}=\tau \cap B_{2}$, and denote by $(B, \tau(s))$ the tangle obtained from $(B, \tau)$ by replacing the subtangle $\left(B_{2}, \tau_{2}\right)$ with a rational tangle of slope $s$ with respect to certain coordinate system on $\partial B_{2}$, set up so that $\tau_{2}$ has slope $\infty$. Let $r_{0}$ be the slope on $\partial N(K)$ which covers a curve of slope 0 on $\partial B_{2}$. Then by the Montesinos trick [17], $\left(V, K, r_{0}-s\right)$ is then the double branched cover of $B$ branched along the tangle $\tau(s)$, and $\left(V, K, r_{0}-s\right)$ is Seifert fibered if and only if $(B, \tau(s))$ is a Montesinos tangle.

To determine the slope $r_{0}$, consider the pretzel surface for the knot $K$ in Figure 2.2(a). It is a once punctured Klein bottle $F$. The boundary of $F$ is the pretzel framing $\lambda$, and one can show that it is a curve of slope 8 on $\partial N(K)$ with respect to the preferred meridian-longitude of $K$. The projection of $F$ is a disk $F^{\prime}$ intersecting the axis at one arc and two individual points, and the boundary of $F^{\prime}$ contains an $\operatorname{arc} \lambda^{\prime}$ on $\partial B_{2}$ which is the projection of the above pretzel framing and will be called the pretzel framing on $\partial B_{2}$. In Figure 2.2(b) $B_{2}$ is the thick dark arc. Its boundary then contains a pair of arcs connecting the 4 branch points, called the blackboard framing. In our case these two framings are actually the same because $F^{\prime}$ has boundary on the blackboard framing except at the two crossings of the dark curve, which contribute -2 and 2 respectively to the pretzel framing and therefore cancel. One can check that the blackboard framing is unchanged under the isotopy from Figure 2.2(b) to Figure 2.2(d) and therefore represents the 0 slope on $\partial B_{2}$. It follows that the pretzel slope 8 is the $r_{0}$ if we set up the coordinates on $\partial B$ in the standard way, ie the horizontal arcs connecting the branch points represent slope 0 and the vertical arcs represent $\infty$. It follows that $(V, K, 8-s)$ is the double branch cover of $(B, \tau(s))$. In particular, $K(7)$ and $K(6)$ are the double branched cover of $(B, \tau(1))$ and $(B, \tau(2))$, respectively.

Since 8 is the pretzel slope, by Example 2.1(2) we see that $(V, K, 8)$ is toroidal. This can be verified as follows. The tangle $\tau(0)$ is shown in Figure 2.2(e), which can be deformed to that in Figure 2.2(f). Note that it has a closed component which bounds a disk $D$ intersecting the other components at two points. The boundary of a regular neighborhood of $D$ is then a Conway sphere, which lifts to the incompressible torus in $K(8)$ bounding a twisted $I$-bundle over the Klein bottle.

The tangle $\tau(1)$ is shown in Figure 2.2(g) and (h). Without fixing the endpoints of the strings on the outside sphere it is equivalent to the $(-1 / 3,-1 / 5)$ Montesinos tangle. Hence its double branched cover $(V, K, 7)$ is a small Seifert fiber space with two singular fibers of indices 3 and 5 , respectively. 
The tangle $\tau(2)$ is shown in Figure 2.2(i), which deforms to that in Figure 2.2(j). There is an obvious Conway sphere bounding a $(1 / 2,-1 / 4)$ Montesinos tangle, and its outside is not a product, hence it lifts to an essential torus in $(V, K, 6)$, bounding a small Seifert fiber space with two singular fibers of indices 2 and 4 respectively.

The following result shows that each pretzel knot of type $(-2,3,2 n+1)$ admits at least two Seifert fibered surgeries, with slopes $6+4 n$ and $7+4 n$. In particular, when $n=3$ it gives the well-known results that 18 and 19 surgeries on the $(-2,3,7)$ pretzel knot are lens spaces; see Fintushel and Stern [5]. Denote by $M\left(r_{1}, r_{2}, r_{3}\right)$ the closed 3 -manifold which is the double branched cover of $S^{3}$ with branch set the Montesinos link $K\left(r_{1}, r_{2}, r_{3}\right)$. To make the statement simple, we do allow $r_{3}=0$ in this theorem, in which case $K\left(r_{1}, r_{2}, r_{3}\right)$ is actually the connected sum of two 2-bridge knots, and $M\left(r_{1}, r_{2}, r_{3}\right)$ is reducible.

Corollary 2.3 Let $K_{n}$ be the $(-2,3,2 n+1)$ pretzel knot in $S^{3}$. Then we have $K_{n}(7+4 n)=M(-1 / 3,3 / 5,1 /(n-2))$ and $K_{n}(6+4 n)=M(1 / 2,-1 / 4,2 /(2 n-5))$. In particular, they are Seifert fibered manifolds for all $n$, except that when $n=2$, $K_{2}(15)=M(-1 / 3,3 / 5,1 / 0)$ is reducible.

Proof Let $r_{n}=r+4 n$, where $r=6,7$. Recall that $K_{n}$ is obtained from $K \subset V \subset S^{3}$ by $n$ right hand full twists along a meridian of $V$, so $K_{n}\left(r_{n}\right)$ is obtained from $(V, K, r)$ by attaching a solid torus $V^{\prime}$ on the outside so that a meridian of $V^{\prime}$ is attached to the curve $\lambda=l_{0}-n m_{0}$. By the Montesinos trick, $K_{n}\left(r_{n}\right)$ is the double branched cover of $S^{3}$ along the link $L$ obtained from $(B, \tau(8-r))$ by attaching a rational tangle $\left(B^{\prime}, \tau^{\prime}\right)$ to the outside of $B$.

To calculate the slope of $\left(B^{\prime}, \tau^{\prime}\right)$, note that $m_{0}$ and $l_{0}$ project to curves of slope $0 / 1$ and $1 / 0$, respectively. One can then check that the curve $\lambda$ projects to a curve $\lambda^{\prime}$ of slope $-1 / n$ on $\partial B$. Since the map $\partial B^{\prime} \rightarrow \partial B$ is orientation reversing, $\lambda^{\prime}$ is of slope $1 / n$ on $\partial B^{\prime}$. We may assume that $\lambda$ has been isotoped to bound a meridian disk $D$ in $V^{\prime}$ which is disjoint from the branch axis. Then $\lambda^{\prime}$ bounds a disk in $B^{\prime}$ disjoint from the tangle strings. It follows that $\left(B^{\prime}, \tau^{\prime}\right)$ is of slope $1 / n$.

For $r=7$, the tangle $(B, \tau(1))$ in Figure 2.2(h) is a Montesinos tangle of length 2, and $K_{n}(7+4 n)$ is the double branched cover of the link obtained by attaching a $1 / n$ tangle to the outside of $(B, \tau(1))$, which one can check is the link $K(-1 / 3,3 / 5,1 /(n-2))$. Hence $K_{n}(7+4 n)=M(-1 / 3,3 / 5,1 /(n-2))$. It is a Seifert fiber space (possibly a lens space) unless $n=2$, which gives the reducible 15 surgery on the $(3,5)$ torus knot. For $r=6$, note the union of $\left(B^{\prime}, \tau^{\prime}\right)$ and the tangle $(B, \tau(2))$ in Figure 2.2(j) is the Montesinos link $K(1 / 2,-1 / 4,2 /(2 n-5))$, thus $K_{n}(6+4 n)=M(1 / 2,-1 / 4,2 /(2 n-5))$, which is a small Seifert fiber space for any $n$. 


\section{Surgery on $K \subset V$ with $\operatorname{wrap}(K)=2$}

Throughout this section we will assume $K \subset V$ is a hyperbolic knot with $\operatorname{wrap}(K)=2$, intersecting a meridian disk $D$ of $V$ twice. Recall that $Y=N(D \cup K),(B, \tau)$ is the tangle obtained by cutting $(V, K)$ along $D$ and $X=V-\operatorname{Int}(Y)=B-\operatorname{Int} N(\tau)$. Let $r$ be a nontrivial slope such that $(V, K, r)$ is nonhyperbolic. Denote by $K_{r}$ the dual knot in $(V, K, r)$ and $(Y, K, r)$.

Lemma 3.1 Suppose $\operatorname{wrap}(K)=2$ and $\partial_{h} X$ is incompressible in $X$. If $X$ is an $I$-bundle with $\partial_{h} X$ the $\partial I$-bundle then $K$ is the Whitehead knot in $V$.

Proof If wind $(K)=0$ then $\partial_{h} X$ is the disjoint union of two copies of a once punctured torus. Hence the hypothesis above implies that $X$ is a product $Q \times I$, where $Q$ is a once punctured torus, and $\partial_{v} X=\partial Q \times I$. Recall that $X=B-\operatorname{Int} N(\tau)$. Let $\tau_{1}, \tau_{2}$ be the two strands of $\tau$. Adding $N\left(\tau_{1}\right)$ to $X$ produces a $D \times I$ with a 1 -handle $H_{1}$ attached to $D \times 1$, and $\tau_{1}$ is the core of $H_{1}$. Similarly $N\left(\tau_{2}\right)$ can be considered as a 2-handle attached to the solid torus $X \cup N\left(\tau_{1}\right)$. Since the result is a 3 -ball, the core of the 2-handle $N\left(\tau_{2}\right)$ intersects the meridian of $X \cup N\left(\tau_{1}\right)$ at a single point. It is now clear that $\tau=\tau_{1} \cup \tau_{2}$ is the tangle shown in Figure 2.1(b), hence $K$ is a Whitehead knot in $V$.

If $\operatorname{wind}(K)=2$ then $\partial_{h} X$ is a twice punctured torus, hence if $X$ is an $I$-bundle then it must be a twisted $I$-bundle over a once punctured Klein bottle $P$, so we can properly embed $P$ in $X \subset B \subset S^{3}$. This is impossible because the union of $P$ and a disk on $\partial B$ would be a closed Klein bottle embedded in $B^{3}$.

An isotopy class $[\alpha]$ of a nontrivial simple closed curve $\alpha$ on $\partial_{h} X$ is called an annular slope if $\alpha$ is not parallel to a boundary component on the surface $\partial_{h} X$, and there is an $h$-essential annulus $A$ in $X$ with $\alpha$ as a boundary component. Note that it is possible that the other boundary component of $A$ could be a boundary parallel curve on $\partial_{h} X$ and hence would not be an annular slope.

Lemma 3.2 Suppose $\partial_{h} X$ is incompressible and $X$ is not an $I$-bundle. Then there is a nonseparating curve $\alpha$ on each component of $\partial_{h} X$ which is disjoint from any annular slope of $\partial_{h} X$ up to isotopy.

Proof Let $\left(W, \partial_{h} W\right)$ be the characteristic pair of the pair $\left(X, \partial_{h} X\right)$, as defined by Jaco and Shalen in [16]. Then $\partial_{h} W=W \cap \partial_{h} X$ is a subsurface of $\partial_{h} X$, and each boundary component of $\partial_{h} W$ is a nontrivial curve on $\partial_{h} X$. By the definition of characteristic pair, $\partial_{h} W$ contains all annular slopes on $\partial_{h} X$ up to isotopy. 
First assume wind $(K)=0$, so each component $F$ of $\partial_{h} X$ is a once punctured torus. It is easy to see that if the result is false then some component $G$ of $\partial_{h} W \cap F$ is full in the sense that $F-G$ is in a collar of $\partial F$; hence it is a once punctured torus. Let $W_{0}$ be the component of $W$ containing $G$. Since $G$ is not a double cover of any other surface, $W_{0}$ must be a trivial $I$-bundle, so $\partial_{h} W_{0}-G$ is also a once punctured torus, which must be on $\partial_{h} X-F$. By Lemma $3.1 X$ is not an $I$-bundle, hence $A=\partial_{v} W_{0}$ is an essential annulus in $X$, cutting off a compact 3-manifold $M$ with $\partial M$ a single torus. Since $X$ is atoroidal and $\partial_{h} X$ is incompressible, we see that $\partial M$ must be compressible inside of $M$, so $X$ being irreducible (since $K$ is hyperbolic) implies that $M$ is a solid torus. Since $X$ is not an $I$-bundle, we see that $A$ runs at least twice along the longitude of $M$. It follows that the union of $A$ and an annulus in $Y$ parallel to $\partial_{v} Y$ forms an essential torus in $V-\operatorname{Int} N(K)$, contradicting the assumption that $K$ is a hyperbolic knot in $V$.

Now assume wind $(K)=2$. Then $\partial_{h} X$ is a twice punctured torus, so if $\partial_{h} X-\partial_{h} W$ does not contain a nonseparating curve then some component $G$ of $\partial_{h} W$ is a once or twice punctured torus. Let $W_{0}$ be the component of $W$ containing $G$. Since $\partial_{h} X$ has genus one, there is no room for another copy of $G$, hence $W_{0}$ must be a twisted $I$-bundle over a once punctured Klein bottle $R$. In particular, $\partial_{h} W_{0}$ must be a twice punctured torus, so $\partial_{h} X-\partial_{h} W_{0}$ is a pair of annuli. We can then extend an embedding of $R$ in $W_{0}$ to an embedding of $R$ in $X$ with $\partial R \subset \partial_{v} X \subset \partial B$. The union of $R$ with a disk on the boundary of $B$ would then be a closed Klein bottle embedded in the 3-ball $B$, which is impossible.

Lemma 3.3 Suppose $K \subset V$ is a hyperbolic knot with $\operatorname{wrap}(K)=2$. Then

(1) $\partial_{h} Y$ is incompressible in $(Y, K, r)$ for all nontrivial $r$;

(2) if $r$ is an integral slope then $(Y, K, r)$ is an $I$-bundle with $\partial_{v} Y$ as its vertical surface;

(3) if $r$ is a nontrivial nonintegral slope then any $h$-essential annulus $Q$ in $(Y, K, r)$ can be isotoped to be disjoint from the dual knot $K_{r}$.

Proof Recall that $Y=N(D \cup K)$, where $D$ is a meridian disk of $V$ intersecting $K$ twice. Let $D_{1}$ be a meridian disk of $K$ in $Y$ with $\partial D_{1} \subset \partial_{h} Y$, and let $Y_{1}=N_{1}\left(D_{1} \cup K\right)$ be a smaller regular neighborhood of $D_{1} \cup K$ such that $Y_{1} \cap \partial Y=\partial D_{1} \times I$. Then the frontier of $Y_{1}$ is an annulus $A$, cutting $Y$ into $Y_{1}$ and another manifold $W$. When $\operatorname{wind}(K)=0, W$ is a product $T_{1} \times I$, where $T_{1}$ is a once punctured torus; when $\operatorname{wind}(K)=2$, the manifold $W$ is a twisted $I$-bundle over a Klein bottle. In either case $W$ is an $I$-bundle with $\partial_{v} Y$ as its vertical boundary. Note that $\partial_{h} W$ is incompressible, and $A$ is an annulus on $\partial_{h} W$, which is incompressible in $W$. 
It is clear that $Y_{1}$ is a solid torus with $K$ as a core, hence $V^{\prime}=\left(Y_{1}, K, r\right)$ is a solid torus for all $r$. When $r$ is an integral slope, $A$ runs along the longitude of $V^{\prime}$ once, hence $(Y, K, r)=W \cup_{A}\left(Y_{1}, K, r\right)$ is homeomorphic to the $I$-bundle $W$ with $\partial_{v} Y$ preserved. When $r$ is a nontrivial nonintegral slope, $A$ runs along the longitude of $V^{\prime}$ more than once. By a standard innermost circle outermost arc argument one can show that $\partial_{h} Y$ is incompressible in $(Y, K, r)$.

If $Q$ is an $h$-essential annulus in $(Y, K, r)$ then it can be isotoped so that $Q \cap A$ has no arc component, so $Q \cap\left(Y_{1}, K, v\right)$ is a set of incompressible annuli, which can then be isotoped to be disjoint from $K_{r}$.

Lemma 3.4 Suppose $K$ is a hyperbolic knot in $V$ with $\operatorname{wrap}(K)=2, K$ is not the Whitehead knot, and $\partial_{h} X$ is incompressible in $X$. Then

(1) $(V, K, r)$ is irreducible, $\partial$-irreducible, and is not Seifert fibered;

(2) if $r$ is not in integral slope then $(V, K, r)$ is hyperbolic.

Proof (1) The irreducibility and $\partial$-irreducibility follows from [1; 8; 19]. It also follows from Lemma 3.3 because $\partial_{h} X$ is an essential surface in $(V, K, r)$ and there is no reducing sphere or compressing disk of $\partial V$ disjoint from $\partial_{h} X$.

Suppose $(V, K, r)$ is Seifert fibered. By Waldhausen [20], any incompressible surface in a Seifert fibered space is either horizontal or vertical. Since $\partial_{h} X$ is not an annulus or torus, it must be horizontal, so both $X$ and $Y(r)$ are $I$-bundles with $\partial_{h} X=\partial_{h} Y$ as their horizontal surface. On the other hand, by Lemma 3.1 this is impossible unless $K$ is the Whitehead knot in $V$, which has been excluded.

(2) If $T$ is an essential torus in $(V, K, r)$ then it must intersect $\partial_{h} X$ because both $(Y, K, r)$ and $X$ are atoroidal. Using a standard cut and paste argument one can show that $T$ can be isotoped so that each component of $T \cap X$ and $T \cap(Y, K, r)$ is an $h$-essential annulus. If $r$ is not an integral slope then by Lemma 3.3(3) the annuli $T \cap(Y, K, r)$ can be isotoped to be disjoint from $K_{r}$, so $T$ would be an essential torus in $V-K$, contradicting the assumption that $K$ is hyperbolic.

A curve $\alpha$ on a surface $F$ is orientation preserving if the orientation of $F$ does not change when traveling through $\alpha$. Alternatively, $\alpha$ is orientation preserving if its regular neighborhood is an annulus, not a Möbius band.

Lemma 3.5 Up to isotopy there are exactly two orientation preserving essential simple closed curves on a Klein bottle $F$. 
Proof Let $\alpha$ be a curve cutting $F$ into an annulus $A$. Suppose $\beta$ is another orientation preserving essential simple closed curve, which intersects $\alpha$ minimally but is not isotopic to $\alpha$. Then $\alpha$ cuts $\beta$ into a set of essential $\operatorname{arcs} C$ on $A$. One can show that $C$ has exactly two components as otherwise $\beta$ would either be orientation reversing (if $|\beta \cap \alpha|$ is odd) or contain more than one component (if $|\beta \cap \alpha|>2$ ). Therefore any other such curve is obtained from $\beta$ by Dehn twists along $\alpha$. It is easy to check Dehn twisting $\beta$ once along $\alpha$ produces a curve isotopic to $\beta$, hence the result follows.

We note that the two curves $\alpha, \beta$ in the above proof are essentially different as $\alpha$ cuts $F$ into an annulus while $\beta$ cuts $F$ into two Möbius bands. Define an orientation preserving essential simple closed curve $\gamma$ on a surface $F$ to be of type I or type II according to whether $F \mid \gamma$ is orientable or not. Thus the curve $\alpha$ above is of type I and $\beta$ of type II. If $C$ is an annular slope of a twisted $I$-bundle $W$ over $F$ and $\varphi: W \rightarrow F$ the $I$-fibration, then up to isotopy $C$ is a boundary component of a vertical annulus. Hence we can define $C$ to be of type I or type II according to whether $\varphi(C)$ is of type I or II on $F$.

Given a compact surface $F$, denote by $\widehat{F}$ the closed surface obtained from $F$ by capping off each boundary component with a disk. Two curves $C_{1}, C_{2}$ on $F$ are weakly equivalent, denoted by $C_{1} \sim C_{2}$, if they are isotopic on $\widehat{F}$.

Let $W$ be a twisted $I$-bundle over a once punctured Klein bottle $R$. Let $\widehat{W}$ be the manifold obtained by attaching a 2-handle to $W$ along $\partial_{v} W$. Then $\widehat{W}$ is a twisted $I$-bundle over the Klein bottle $\widehat{R}$. Denote by $\varphi: \partial_{h} W \rightarrow \partial \widehat{W}$ the inclusion map. Let $C_{1}$ be a type I annular slope on $\partial_{h} W$. There are infinitely many annular slopes on $F$ that intersect $C_{1}$ essentially, but the following shows that these are all weakly equivalent.

Denote by $I\left(C_{1}, C_{2}\right)$ the algebraic intersection number between two curves $C_{1}, C_{2}$, which is well defined up to sign on any orientable surfaces.

Lemma 3.6 Let $C_{1}$ be a type I annular slope on $\partial_{h} W$. Then there is a type II annular slope $C_{2}$ on $\partial_{h} W$ intersecting $C_{1}$ at a single point, such that if $C_{3}$ is an annular slope on $\partial_{h} W$ then it is either weakly equivalent to $C_{2}$, or isotopic on $\partial_{h} W$ to a curve disjoint from $C_{1}$. In particular, $I\left(C_{3}, C_{i}\right)=0$ for some $i=1,2$.

Proof Fix $I$-bundle structures of $W$ and $\widehat{W}$ and let $\rho: W \rightarrow R$ and $\hat{\rho}: \widehat{W} \rightarrow \widehat{R}$ be the projection maps. We may assume that $C_{1}$ is a boundary component of a vertical annulus $A_{1}$. Then $\rho\left(A_{1}\right)=\rho\left(C_{1}\right)=\alpha_{1}$ is a type I curve on $R$. Let $\alpha_{2}$ be a type II curve on $R$ intersecting $\alpha_{1}$ minimally at two points as given in the proof of Lemma 3.5, and let $C_{2}$ be a boundary component of $\rho^{-1}\left(\alpha_{2}\right)$. The two intersection points of 
$\alpha_{1} \cap \alpha_{2}$ lift to two points of $C_{1} \cap \rho^{-1}\left(\alpha_{2}\right)$, one on each component of $\rho^{-1}\left(\alpha_{2}\right)$. Hence $C_{1}$ intersects $C_{2}$ at a single point.

Assume $C_{3}$ is another annular slope on $F$. We may assume it is a boundary component of a vertical annulus $A_{3}$, so $\alpha_{3}=\rho\left(A_{3}\right)$ is an orientation preserving essential simple closed curve on $R$. By Lemma $3.5 \alpha_{3}$ is isotopic to either $\alpha_{1}$ or $\alpha_{2}$ on $\widehat{R}$. Cutting $R$ along $\alpha_{1}$ produces a once punctured annulus, hence it is easy to see that if $\alpha_{3}$ is isotopic to $\alpha_{1}$ on $\widehat{R}$ then it is also isotopic to $\alpha_{1}$ on $R$, in which case $C_{3}$ can be isotoped to be disjoint from $C_{1}$. If $\alpha_{3}$ is isotopic to $\alpha_{2}$ then $C_{3}$ is isotopic to a component of $\hat{\rho}^{-1}\left(\alpha_{2}\right)$ on $\partial \widehat{W}$. Since the two components of $\hat{\rho}^{-1}\left(\alpha_{2}\right)$ are parallel to each other, we see that $C_{3}$ is isotopic to $C_{2}$ on $\partial \widehat{W}$ and hence is weakly equivalent to $C_{2}$ on $\partial W$.

Lemma 3.7 Suppose wind $(K)=2$. Let $r$ be an integral slope and $K_{r}$ the dual knot in $(Y, K, r)$. Let $\alpha$ be a simple closed curve on $\partial_{h} Y$ which is isotopic to $K_{r}$ in $(Y, K, r)$, let $\beta$ be an annular slope on $\partial_{h} Y$ intersecting $\alpha$ essentially at one point, as given in Lemma 3.6. Suppose $s \neq r$ is another integral slope on $\partial N(K)$. Then $\beta$ is not weakly equivalent to an annular slope of $\partial_{h} Y$ in $(Y, K, s)$.

Proof By the proof of Lemma 3.3, $(Y, K, r)$ is obtained from an $I$-bundle $W$ over $R$ by attaching a solid torus $V^{\prime}$ along a longitudinal annulus of $V^{\prime}$, and $K_{r}$ is the core of $V^{\prime}$. It is easy to see that $\alpha$ is a type $I$ annular slope. The identity map of $W$ extends to a homeomorphism $\psi:(Y, K, s) \rightarrow(Y, K, r)$, and the restriction of $\psi$ on $\partial Y$ is a Dehn twist $\tau_{\alpha}^{n}$ along $\alpha$, where $n=s-r \neq 0$. In particular, the curve $\beta$ is mapped to $\beta^{\prime}=\tau_{\alpha}^{n}(\beta)$ on $\partial_{h}(Y, K, r)$. We have $\left|I\left(\beta^{\prime}, \alpha\right)\right|=1$ and $\left|I\left(\beta^{\prime}, \beta\right)\right|=|n| \neq 0$; hence by Lemma $3.6 \beta^{\prime}$ is not weakly equivalent to an annular slope of $\partial_{h}(Y, K, r)$. Since the homeomorphism $\psi:(Y, K, s) \rightarrow(Y, K, r)$ maps $\beta$ to $\beta^{\prime}$, it follows that $\beta$ is not weakly equivalent to an annular slope of $\partial_{h}(Y, K, s)$.

Theorem 3.8 Suppose $K$ is a hyperbolic knot with wrap $(K)=2$ in a solid torus $V, K$ is not the Whitehead knot, and $\partial_{h} X$ is incompressible in $X$. Then $K$ admits at most one exceptional surgery $(V, K, r)$, which must be a toroidal surgery and $r$ an integral slope.

Proof By Lemma $3.4(V, K, r)$ is irreducible and not a solid torus or small Seifert fiber space, and it is also atoroidal when $r$ is not an integral slope. Hence we need only show that $K$ admits at most one integral toroidal surgery.

First assume wind $(K)=2$. By Lemma 3.2 there is a nonseparating curve $\gamma$ on $\partial_{h} X$ which is disjoint from all annular slopes of $X$ up to isotopy. Suppose $(V, K, r)$ is 
toroidal. Let $\alpha, \beta$ be the annular slopes on $\partial_{h} Y=\partial_{h}(Y, K, r)$ as given in Lemma 3.7. Let $q: \partial_{h} X \rightarrow \partial_{h} Y$ be the gluing map. We claim that $q(\gamma) \sim \beta$.

Let $T$ be an essential torus in $(Y, K, r)$ intersecting $\partial X$ minimally. Since $\partial_{h} X$ is incompressible, each component of $A_{1}=T \cap X$ and $A_{2}=T \cap(Y, K, r)$ is an $h-$ essential annulus. In particular, each boundary component of $A_{1}$ is either an annular slope of $X$ or boundary parallel, hence by the above we may assume $\gamma \cap \partial A_{1}=\varnothing$, so $q(\gamma) \cap \partial A_{2}=\varnothing$. On the other hand, each boundary component of $A_{2}$ is either parallel to a boundary component of $\partial_{h} Y$, or is an annular slope of $(Y, K, r)$; hence by Lemma 3.6 we may assume that it is either disjoint from $\alpha$ or weakly equivalent to $\beta$. If no component of $\partial A_{2}$ is weakly equivalent to $\beta$ then $\partial A_{2}$ can be isotoped to be disjoint from $\alpha$, hence $T$ can be isotoped to be disjoint from $K_{r}$ because by definition $K_{r}$ is isotopic to $\alpha$. This contradicts the assumption that $K$ is hyperbolic. Therefore at least one component $C$ of $\partial A_{2}$ satisfies $C \sim \beta$; in particular, it is nonseparating. Since $C$ and $q(\gamma)$ are disjoint and they are both nonseparating curves on the punctured torus $\partial_{h} Y$, we have $C \sim q(\gamma)$, hence the claim $q(\gamma) \sim \beta$ follows.

Now if $s$ is another toroidal slope of $K$ then by the above, $\beta \sim q(\gamma)$ is also an annular slope on $\partial_{h}(Y, K, s)$, which contradicts Lemma 3.7, completing the proof for the case of $\operatorname{wind}(K)=2$.

The proof for the case of wind $(K)=0$ is similar. In this case $(Y, K, r)$ is a product $F \times I$. Let $F_{i}=F \times i$ for $i=0,1$. Let $q: \partial_{h} X \rightarrow \partial_{h} Y=F_{0} \cup F_{1}$ be the gluing map, and $G_{i}=q^{-1}\left(F_{i}\right)$. By Lemma 3.2 there is a nonseparating curve $\gamma_{i}$ on $G_{i}$ which is disjoint from all annular slopes of $X$ up to isotopy. Let $\gamma_{i}^{\prime}$ be the curve $q\left(\gamma_{i}\right)$ on $G_{i}$. We claim that $\gamma_{0}^{\prime}, \gamma_{1}^{\prime}$ cobound an annulus and hence is homologous in $(Y, K, r)$.

Let $T$ be an essential torus in $(V, K, r)$. As above, the hyperbolicity of $K$ implies that there is a component $A^{\prime}$ of $A_{1}=T \cap(Y, K, r)$ which cannot be isotoped off $K_{r}$. Let $\beta_{i}=A^{\prime} \cap F_{i}$. Since each side of $A^{\prime}$ must be adjacent to an essential annulus in $X$, we see that $q^{-1}\left(\beta_{i}\right)$ is an annulus slope on $G_{i}$. Since $G_{i}$ is a once punctured torus, any annular slope on $G_{i}$ is a nonseparating curve disjoint from $\gamma_{i}$ and therefore must be isotopic to $\gamma_{i}$. If follows that $\gamma_{i}^{\prime}$ is isotopic to $\beta_{i}$ on $F_{i}$, hence $A^{\prime}$ can be isotoped to have $\partial A^{\prime}=\gamma_{0}^{\prime} \cup \gamma_{1}^{\prime}$, and the claim follows.

For the same reason, if $s$ is another integral toroidal slope of $K$ then $\gamma_{0}^{\prime}$ and $\gamma_{1}^{\prime}$ must also be homologous in $(Y, K, s)$. On the other hand, as in the proof of Lemma 3.7, there is a homeomorphism $\psi:(Y, K, r) \rightarrow(Y, K, s)$ which is the identity map on $F_{0}$ and the Dehn twist map $\tau_{\alpha}^{n}$ on $F_{1}$, where $n=r-s \neq 0$ and $\alpha$ is the curve on $F_{1}$ isotopic to $K_{r}$ in $(Y, K, r)$. Since $A^{\prime}$ cannot be isotoped off $K_{r}, \gamma_{1}^{\prime}$ has essential intersection with $\alpha$, hence $\psi\left(\gamma_{1}^{\prime}\right)$ is not homologous to $\gamma_{0}^{\prime}$ in $(Y, K, s)$ if $s \neq r$, a contradiction. 


\section{Surgery on $K_{n}$}

As in Section 1, define $K_{n}=\varphi_{n}(K)$ and $r_{n}=\varphi_{n}(r)$, where $\varphi_{n}: V \rightarrow S^{3}$ is the composition of the standard embedding of $V$ into $S^{3}$ with $n$ full right hand twists along a meridian disk of $V$, and $r$ is a slope of $K$. If $(V, K, r)$ is a small Seifert fiber manifold then $K_{n}\left(r_{n}\right)$ is either small Seifert fibered or reducible, hence is always nonhyperbolic. In general, if $(V, K, r)$ is toroidal then it is possible that $K_{n}\left(r_{n}\right)$ may be hyperbolic for infinitely many $n$; however, this will not happen if $\operatorname{wind}(K)=2$. The main result of this section shows that if $\operatorname{wrap}(K)=2$ and $(V, K, r)$ is toroidal then either $K_{n}\left(r_{n}\right)$ is toroidal for all but at most three $n$, or it is atoroidal and nonhyperbolic for all $n$. In particular, it can be hyperbolic for at most three $n$; see Theorem 4.3 for more details.

Let $D$ be a meridional disk of $V$ with $n_{1}=|D \cap K|=2$, and $T$ an essential torus in $(V, K, r)$ such that $n=n_{2}=\left|T \cap K_{r}\right|$ is minimal. Let $E(K)$ be the knot exterior $V-\operatorname{Int} N(K)$. Denote by $Q_{1}$ the punctured disk $D \cap E(K)$, and by $Q_{2}$ the punctured torus $T \cap E(K)$. Considering the disks $D \cap N(K)$ and $T \cap N\left(K_{r}\right)$ as fat vertices, and the arc components of $Q_{1} \cap Q_{2}$ as edges, we obtain graphs $\Gamma_{1}, \Gamma_{2}$ on $D$ and $T$, respectively, with $n_{i}$ vertices on $\Gamma_{i}$. Denote by $m$ the meridional slope of $K$, and by $\Delta=\Delta(m, r)$ the distance (ie the geometric intersection number) between $m$ and $r$. By [11] we have $\Delta \leq 2$. Each boundary component of $Q_{1}$ intersects each component of $Q_{2}$ at $\Delta$ points; hence each vertex of $\Gamma_{1}$ has valence $n \Delta$, and each vertex of $\Gamma_{2}$ has valence $n_{1} \Delta=2 \Delta$.

This describes the standard way of creating useful graphs from the intersection pattern of surfaces, before and after doing Dehn surgery. We refer the readers to Culler, Gordon, Luecke and Shalen [4] and also [12] for standard terms and basic results related to intersection graphs, such as Scharlemann cycles, extended Scharlemann cycles, and signs of vertices. In particular, the minimality of $n$ and $\operatorname{wrap}(K)=2$ imply that there are no trivial loops in $\Gamma_{i}$, so $\Gamma_{1}$ is a set of $n \Delta$ parallel edges. Each vertex of $\Gamma_{i}$ has a sign. An edge of $\Gamma_{i}$ is a positive edge if the two vertices on its endpoints are of the same sign. There is a one to one correspondence between edges of $\Gamma_{1}$ and $\Gamma_{2}$. The Parity Rule of [4, page 279] says that an edge is positive on one graph if and only if it is negative on the other. If wind $(K)=2$ then we have that both vertices of $\Gamma_{1}$ are positive, and hence all edges on $\Gamma_{1}$ are positive edges, and all edges on $\Gamma_{2}$ are negative edges. Similarly if wind $(K)=0$ then we have that all edges of $\Gamma_{1}$ are negative and all edges of $\Gamma_{2}$ are positive. 
Lemma 4.1 Suppose $K \subset V$ has $\operatorname{wind}(K)=\operatorname{wrap}(K)=2$. If $(V, K, r)$ is toroidal then it contains an essential torus $T$ such that

(1) if $n>2$ then $\Gamma_{1}$ has no extended Scharlemann cycle;

(2) $n=2$ or 4 ;

(3) $\Delta=1$;

(4) $T$ bounds a small Seifert fiber space.

Proof (1) This is found in Boyer and Zhang [2, Lemma 2.9] or Gordon and Luecke [10, Theorem 3.2]. An extended Scharlemann cycle can be used to find an essential torus in $(V, K, r)$ which has fewer intersections with $K_{r}$, contradicting the minimality of $n$.

(2) The parity rule implies that $n$ must be even as otherwise there would be an edge in $\Gamma_{1}$ with the same label on its two endpoints, so it would be a positive edge on both graphs. If $n>4$ then the $n$ parallel positive edges of $\Gamma_{1}$ contain an extended Scharlemann cycle, contradicting (1); see the author [25, Lemma 1.4].

(3) Assume $\Delta \geq 2$. If $n=4$ then $\Gamma_{1}$ has an extended Scharlemann cycle, a contradiction. Hence we may assume $n=2$. By Gordon [9, Lemma 2.1], no two edges can be parallel on both graphs, so we must have $\Delta=2$, and the four edges of $\Gamma_{2}$ are mutually nonparallel on $\Gamma_{2}$. A disk face $E$ of $\Gamma_{2}$ then has four edges. Now cut $V$ along $D$, let $D_{1}, D_{2}$ be the two copies of $D$ on $B=V \mid D$, and let $\tau=\tau_{1} \cup \tau_{2}=K \cap B$ be the two strings of $K$ in $B$. Then the neighborhood of $D_{1} \cup D_{2} \cup \tau_{1} \cup \tau_{2}$ is a solid torus $V^{\prime}$ in $B$. The boundary curve of $E$ runs four times along $\tau$, twice along each $\tau_{i}$. Since all segments of $\partial E$ on $D$ and $\partial N(\tau)$ are essential arcs, we see that $\partial E$ intersects a meridian of $\tau_{i}$ twice in the same direction, hence $V^{\prime} \cup N(E)$ ) is a punctured projective space in the 3-ball $B$, which is impossible.

(4) We now have $\Delta=1$ and $n=2$ or 4 . The edges of $\Gamma_{1}$ form one or two Scharlemann cycles, according to whether $n=2$ or 4 ; see Figure 4.1. By [4, Lemma 2.5.2] the essential torus $T$ is separating in $(V, K, r)$. It cuts $(V, K, r)$ into two regions; the one containing $\partial V$ is called the white region, and the other one the green region. From Figure 4.1 we can see that the Scharlemann disk $G$ bounded by a Scharlemann cycle $e_{1} \cup e_{2}$ is in the green region since there is no extended Scharlemann cycle. When shrinking each fat vertex of $\gamma_{2}$ to a point, $e_{1} \cup e_{2}$ becomes a loop on $T$, which must be essential by [2, Lemma 2.8]. Let $H$ be the part of $N\left(K_{r}\right)$ in the green region. Then $N(T \cup H \cup G)$ has two torus boundary components, and the one $T^{\prime}$ inside the green region has fewer intersections with $K_{r}$. By the choice of $T$, this $T^{\prime}$ must bound a solid torus $V^{\prime}$. The green region is now the union of two solid tori $V^{\prime}$ and $V^{\prime \prime}=N(H \cup E)$ 
with $V^{\prime} \cap V^{\prime \prime}$ an annulus, hence $T$ being essential implies that $V^{\prime} \cup V^{\prime \prime}$ is a small Seifert fiber space bounded by $T$, whose orbifold is a disk with two cone points.

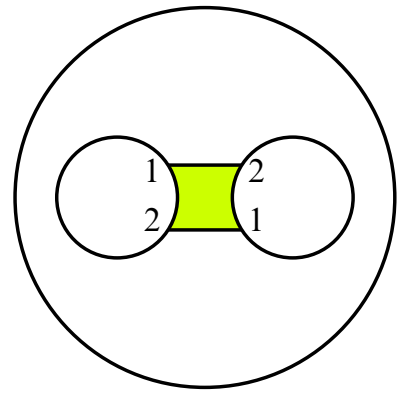

(a)

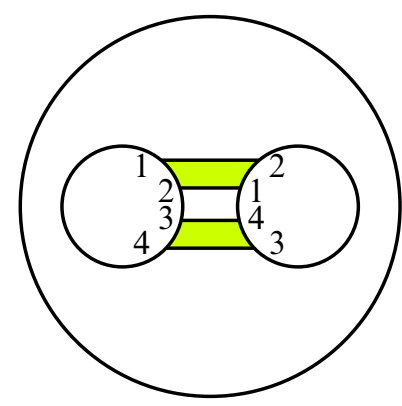

(b)

Figure 4.1

Lemma 4.2 Suppose $K \subset V$ has $\operatorname{wrap}(K)=2$. If $(V, K, r)$ is toroidal then $\Delta=1$, and it contains an essential torus $T$ such that either $T$ is nonseparating or it bounds a small Seifert fiber space.

Proof The conclusion holds if $(V, K, r)$ contains a nonseparating torus, so we may assume that all essential tori in $(V, K, r)$ are separating. If $(V, K, r)$ contains a Klein bottle $F$ then $T=\partial N(F)$ must be incompressible as otherwise $(V, K, r)$ would be reducible. $T$ is also not parallel to $\partial V$, otherwise $(V, K, r)=N(F)$ would be atoroidal. Hence $T$ is an essential torus bounding the small Seifert fiber space $N(F)$ and the result follows. Therefore we may also assume that $(V, K, r)$ contains no Klein bottle.

The case $\operatorname{wind}(K)=2$ is covered by Lemma 4.1, so we assume $\operatorname{wind}(K)=0$. The two vertices of $\Gamma_{1}$ on $D$ are now antiparallel, so all edges of $\Gamma_{1}$ are negative. By Gordon and the author [14, Lemma 2.3(1)], if $\Gamma_{1}$ has more than $n$ parallel negative edges then $T$ would be nonseparating, contradicting the assumption above. Hence we must have $\Delta=1$.

By the proof of [14, Lemma 2.2(3)], the $n$ edges form mutually disjoint essential cycles of equal length on $\Gamma_{2}$. All vertices on the same cycle are parallel; since $T$ is separating, the number of positive vertices is equal to that of negative vertices, hence we have an even number of cycles.

On the twice punctured disk $D \cap X$, the edges $e_{1}, \ldots, e_{n}$ cut it into one annulus and $n-1$ rectangles $D_{1}, \ldots, D_{2 n-1}$. As before, call the two components of $(V, K, r) \mid T$ the white region $W$ and the green region $G$, with the white region containing $\partial V$. 
Then $n / 2$ of the rectangles $D_{2 i-1}$ are in the green region. Also, the Dehn filling solid torus $N\left(K_{r}\right)$ is cut by $T$ into $n$ components $H_{1}, \ldots, H_{n}$, with $H_{2 i-1}$ in the green region, and each $H_{2 i-1}$ is incident to two of the rectangles. It follows that if we shrink each $H_{2 i-1}$ to an $\operatorname{arc} \alpha_{i}$ then $\bigcup\left(H_{2 i-1} \cup D_{2 i-1}\right)$ becomes a set of annuli or Möbius bands containing these $\alpha_{i}$, with boundary on the above cycle. But since the two ends of $\alpha_{i}$ are of opposite signs, which by the above are on different cycles, we see that there is no Möbius band in the above union, so they are all annuli.

Let $A$ be one of these annuli. Then $\partial A$ cuts $T$ into two annuli $A_{1}, A_{2}$. Since we assumed that $(V, K, r)$ contains no Klein bottle, each $A \cup A_{i}$ is a torus instead of Klein bottle, hence the frontier of $N(T \cup A)$ consists of three tori $T_{0} \cup T_{1} \cup T_{2}$, with $T_{1}, T_{2}$ in the green region. One can check that each $T_{i}$ has fewer intersections with $K_{r}$ than $T$, hence by the minimality of $n$ we see that each $T_{i}$ bounds a solid torus $V_{i}$, which must be disjoint from $T \cup A$ as otherwise it would contain $\partial V$ and hence have at least two boundary components, contradicting the assumption that $V_{i}$ are solid tori. It now follows that $G$ is homeomorphic to the manifold obtained by gluing $V_{1}, V_{2}$ along an annulus. The incompressibility of $T$ then implies that $G$ is a small Seifert fiber space with orbifold a disk with two cone points.

Theorem 4.3 Suppose $\operatorname{wrap}(K)=2$, and $(V, K, r)$ is nonhyperbolic. Then $K_{n}\left(r_{n}\right)$ is nonhyperbolic for all but at most three $n$. Moreover, one of the following holds:

(1) there is an $n_{0}$ such that $K_{n}\left(r_{n}\right)$ is toroidal unless $\left|n-n_{0}\right| \leq 1$;

(2) $K_{n}\left(r_{n}\right)$ is atoroidal for all $n$, and there exist $q_{1}, q_{2} \in \mathbb{Z}$ such each $K_{n}\left(r_{n}\right)$ is either reducible or has a small Seifert fibration with $q_{1}, q_{2}$ as the indices of two of its singular fibers.

Proof By $[1 ; 19](V, K, r)$ is irreducible and not a solid torus, hence it is either a small Seifert fibered manifold or toroidal. If it is a small Seifert fibered manifold then (2) holds, where $q_{1}, q_{2}$ are the indices of the two singular fibers of $(V, K, r)$.

Suppose $(V, K, r)$ is toroidal. Let $T$ be an essential torus of $(V, K, r)$ given by Lemma 4.2. Note that $K_{n}$ is obtained by Dehn filling on one component of a hyperbolic link, hence by the author [22] it is nontrivial for all but at most two adjacent integers $n$. By Gabai [6], $K_{n}\left(r_{n}\right)$ cannot contain a nonseparating sphere if $K_{n}$ is nontrivial. Therefore if $T$ is nonseparating then it remains a nonseparating incompressible torus in $K_{n}\left(r_{n}\right)$ for all but at most two consecutive $n$, hence (1) holds.

We may now assume $T$ is separating. By Lemma 4.2, $T$ cuts $(V, K, r)$ into $M_{1}, M_{2}$, where $M_{2}$ contains $T_{0}=\partial V$, and $M_{1}$ is a small Seifert fiber space. Thus we have $K_{n}\left(r_{n}\right)=M_{1} \cup_{T} M_{2}\left(r_{n}\right)$. Let $q_{1}, q_{2}$ be the indices of the singular fibers of $M_{1}$. 
By [4, Theorem 2.4.4], if $M_{2}$ is not a cable space then $T$ is incompressible in $M_{2}\left(r_{n}\right)$ and hence incompressible in $K_{n}\left(r_{n}\right)$, for all but at most two consecutive $n$, so again (1) follows and we are done.

We now assume that $M_{2}$ is a cable space. Let $A$ be an essential annulus in $M_{2}$ with one boundary component on each of $T$ and $T_{0}$, and let $\gamma_{0}$ be the boundary slope of $A$ on $T_{0}$. Let $(m, l)$ be a meridian-longitude pair of $T_{0}=\partial V$. Then $K_{n}\left(r_{n}\right)$ is obtained from $(V, K, r)$ by Dehn filling on $T_{0}$ along the slope $\alpha_{n}=l-n m$.

By [4, Theorem 2.4.3], there is a slope $\gamma_{0}$ on $T_{0}$ such that $T$ remains incompressible in $M_{2}\left(\alpha_{n}\right)$ if $\Delta\left(\alpha_{n}, \gamma_{0}\right) \geq 2$. If $m \neq \gamma_{0}$ then at most three consecutive $\alpha_{n}$ satisfy the above condition and hence (1) holds. Now assume $m=\gamma_{0}$. Then $M_{2}\left(\alpha_{n}\right)$ is a solid torus for all $n$, so $K_{n}\left(r_{n}\right)$ is the union of the small Seifert fiber space $M_{1}$ and the solid torus $M_{2}\left(\alpha_{n}\right)$. If the fiber of $M_{1}$ is the meridional slope of $M_{2}\left(\alpha_{n}\right)$ then $K_{n}\left(r_{n}\right)$ is reducible, and if not then the Seifert fibration of $M_{1}$ extends to a small Seifert fiber structure of $K_{n}\left(r_{n}\right)$. Hence (2) holds in this case.

\section{Surgery on wrapped Montesinos knots}

Denote by $T\left[t_{1}, \ldots, t_{p}\right]$ the Montesinos tangle consisting of $p$ rational tangles of slopes $t_{i}$; see Figure 5.1(a) for $p=2$, where a circle with label $t_{i}$ represents a rational tangle of slope $t_{i} \neq 1 / 0$. Up to isotopy we may assume $t_{i}$ are not integers unless $p=1$. We can add two strings to connect the top endpoints to the bottom ones to make it a knot of wrapping number 2 in a solid torus $V$. Up to homeomorphism of $V$ there are two ways to add these two strings, as shown in Figure 5.1(b)-(c), denoted by $K^{0}\left(t_{1}, \ldots, t_{p}\right)$ and $K^{1}\left(t_{1}, \ldots, t_{p}\right)$, respectively. Recall that the circle with a dot in these figures represents the component $K^{\prime}$ to be removed, so $V=$ $S^{3}-\operatorname{Int} N\left(K^{\prime}\right)$. Only one of these is a knot if the two top endpoints of the tangle belong to different strings. We call these knots wrapped Montesinos knots in solid tori. Note that if $K=K^{a}\left(t_{1}, \ldots, t_{p}\right)$ for $a=0,1$ then $K_{n}$ is a Montesinos knot $M\left(1 /(a+2 n), t_{1}, \ldots, t_{p}\right)=M\left(t_{1}, \ldots, t_{p}, 1 /(a+2 n)\right)$ in $S^{3}$. The purpose of this section is to determine all exceptional Dehn surgeries on these wrapped Montesinos knots in solid tori.

As before, we fix a meridian-longitude pair $\left(m_{0}, l_{0}\right)$ of $K \subset V$ so that it becomes the preferred meridian-longitude pair of $K_{0} \subset S^{3}$. A slope $r$ is then represented by a rational number $u / v$ if it represents $\pm\left(u m_{0}+v l_{0}\right)$ in $H_{1}(\partial N(K))$. We first consider the knot $K=K^{1}(-1 / 2,1 / 3)$. By Proposition 2.2, $K(6)$ and $K(8)$ are toroidal, and $K(7)$ is small Seifert fibered. The following lemma shows that there is no other exceptional surgery on this knot. 


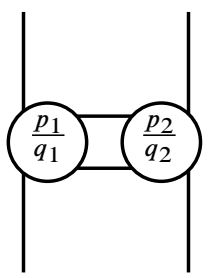

(a)

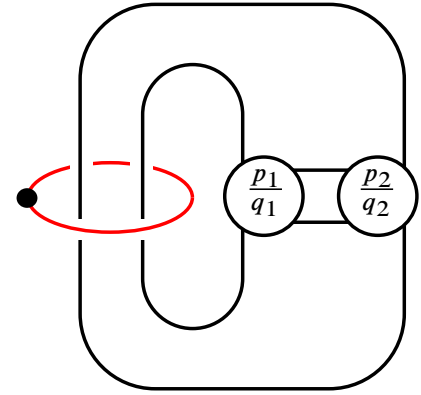

(b)

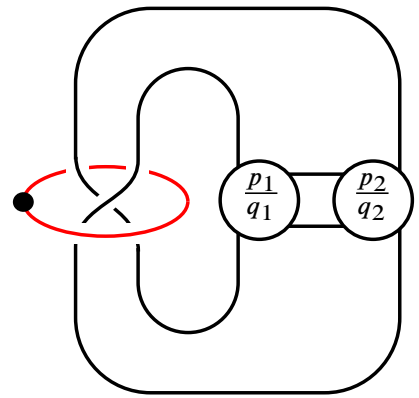

(c)

Figure 5.1

Lemma 5.1 Let $K=K^{1}(-1 / 2,1 / 3)$. Then $(V, K, r)$ is an exceptional surgery if and only if $r=6,7,8$.

Proof Let $\varphi_{n}: V \rightarrow S^{3}$ be the embedding defined in Section 4. Then $K_{n}=\varphi_{n}(K)$ is the $(-2,3,1+2 n)$ pretzel knot, and we have $r_{n}=\varphi_{n}(r)=r+4 n$ with respect to the preferred meridian-longitude of $K_{n}$. Assume that $(V, K, r)$ is nonhyperbolic. By Theorem 4.3 one of the following holds:

(1) $K_{n}\left(r_{n}\right)$ is toroidal for all but at most three $r_{n}$;

(2) $K_{n}\left(r_{n}\right)$ is reducible or small Seifert fibered for all $n$.

If (1) is true then by the author [26] we have $r=8$. Hence we assume (2) holds. When $n=-1, K_{n}$ is the $(-2,3,-1)$ pretzel knot, which can be deformed to the mirror image of the knot $5_{2}$ on the knot table. By [3] it admits only three Seifert fibered surgeries, with slopes $1,2,3$, respectively, and there is no reducible surgery. Since $r_{n}=r+4 n=r-4$ when $n=-1$, we have $r=5,6,7$.

It remains to show $(V, K, 5)$ is hyperbolic. Let $r=5$. The above shows that $K_{n}\left(r_{n}\right)$ is small Seifert fibered for $n=-1$. For $n=0,1,2$, the knot $K_{n}$ is the $(2,5),(3,4)$ and $(3,5)$ torus knot, respectively, hence $K_{n}\left(r_{n}\right)$ cannot be toroidal for these four $n$. By Theorem 4.3, this implies that if $(V, K, 5)$ is nonhyperbolic then conclusion (2) of that theorem must hold, ie there exists $q_{1}, q_{2}$ such that each $K_{n}\left(r_{n}\right)$ is either reducible, or has a small Seifert fibration with $q_{1}, q_{2}$ as the indices of two of its singular fibers.

For $n=0, K_{n}$ is the $(2,5)$ torus knot, and $r_{n}=r=5$. The cabling slope of $K_{0}$ is $2 \times 5=10$, hence the Seifert fiber structure of the exterior of $K_{0}$ extends over the Dehn filling solid torus, whose core is then a singular fiber of index $10-5=5$. Therefore $K_{0}(5)$ is a small Seifert fibered manifold with three singular fibers of indices $2,5,5$, respectively. Similarly, $K_{1}$ is the $(-2,3,3)$ pretzel knot, which is 
the $(3,4)$ torus knot. The cabling slope of $K_{1}$ is $3 \times 4=12$, and the surgery slope is $r_{1}=5+4=9$, so that after Dehn surgery the manifold $K_{1}\left(r_{1}\right)$ is a small Seifert fibered manifold with three singular fibers of indices 3, 4, 3, respectively. We have by Jaco [15, Theorem VI.17] that Seifert fibrations for these manifolds are unique. This is then a contradiction to Theorem 4.3 since no pair of indices of the singular fibers of $K_{0}\left(r_{0}\right)$ match those of $K_{1}\left(r_{1}\right)$.

Lemma 5.2 Let $K=K^{1}(-1 / 2,1 / q)$, where $|q| \geq 3$ is odd. Let $X$ be the tangle space as defined in Section 2. Then $F=\partial_{h} X$ is incompressible unless $q=3$.

Proof Let $\hat{X}$ be the manifold obtained by attaching a 2-handle to $X$ along the annulus $\partial_{v} X$, and let $\hat{F}=\partial \hat{X}$ be the corresponding surface. Note that $X$ is a handlebody of genus 2 , so if $F$ is compressible then there is a nonseparating compressing disk $D_{1}$, which remains a compressing disk in $\hat{X}$. Let $L$ be the link obtained by adding two horizontal arcs to the tangle $T[-1 / 2,1 / q]$. Then $\hat{X}=E(L)$, hence $L$ is a trivial knot. On the other hand, it is easy to see that $L$ is a $(2, q-2)$ torus knot. Since $|q| \geq 3$, it follows that $L$ is trivial if and only if $q=3$.

The knot $K=K^{a}\left(1 / q_{1}, 1 / q_{2}\right)$ in $V$ has an obvious spanning surface which is a once punctured torus or Klein bottle, called the pretzel surface. Its boundary slope is called the pretzel slope of $K$.

Lemma 5.3 Let $K=K^{a}\left(1 / q_{1}, 1 / q_{2}\right)$ be a pretzel knot in $V,\left|q_{i}\right|>1$ and let $\left\{q_{1}, q_{2}\right\} \neq\{\mp 2, \pm 3\}$. Then $(V, K, r)$ is hyperbolic unless $r$ is the pretzel slope.

Proof By Lemma 5.2 and [23, Lemma 3.3] the surface $\partial_{h} X$ is incompressible, hence by Theorem 3.8 we see that the knot $K \subset V$ admits no reducible or Seifert fibered surgery and at most one toroidal surgery. Since the surgery along the pretzel slope $r$ contains either a nonseparating torus or a Klein bottle and hence is nonhyperbolic, it is the only exceptional surgery slope.

Lemma 5.4 Suppose $K=K^{1}(-1 / 2,2 / 5)$. Then $(V, K, r)$ admits no exceptional surgery.

Proof We have $K_{n}=M(-1 / 2,2 / 5,1 /(1+2 n))$. Checking the list in [26, Theorem 1.1], we see that $K_{n}$ admits no toroidal surgery when $n>9$, so by Theorem 4.3, if $(V, K, r)$ is an exceptional surgery then $K_{n}\left(r_{n}\right)$ is atoroidal and nonhyperbolic for all $n$. In particular, this should be true for $n=-1$, in which case we have that $K_{n}=M(-1 / 2,2 / 5,-1)$ can be deformed to the 2-bridge knot associated to the rational number $-1 /(3-1 / 4)=-4 / 11$. On the other hand, by [3] this knot admits only one exceptional surgery, which produces a toroidal manifold. Hence we have a contradiction. 
Two Montesinos tangles $T\left[t_{1}, \ldots, t_{k}\right]$ and $T\left[s_{1}, \ldots, s_{k}\right]$ are equivalent if $s_{i}-t_{i}$ are integers, and $\sum s_{i}=\sum t_{i}$, in which case $K^{a}\left(t_{1}, \ldots, t_{k}\right)$ is isotopic to $K^{a}\left(s_{1}, \ldots, s_{k}\right)$. Any $t_{i}=0$ can be added or deleted without affecting the knot type. Note that $K=K^{a}\left(t_{1}, \ldots, t_{k}\right)$ is isotopic to $K^{\prime}=K^{a}\left(t_{k}, \ldots, t_{1}\right)$, and is the mirror image of $K^{\prime \prime}=K^{a}\left(-t_{1}, \ldots,-t_{k}\right)$, so $(V, K, r)$ is homeomorphic to $\left(V, K^{\prime}, r\right)$ and $\left(V, K^{\prime \prime},-r\right)$. When $k=1$, twisting $m$ times along a meridional disk of $V$ will change $K=K^{a}\left(t_{1}\right)$ to $K^{\prime \prime \prime}=K^{a}\left(1 /\left(2 m+1 / t_{1}\right)\right)$. We will consider these knots $K, K^{\prime}, K^{\prime \prime}, K^{\prime \prime \prime}$ as equivalent. We may assume that $K$ is not equivalent to $K^{a}(0)$ or $K^{a}(1 / q)$ as otherwise $K$ is nonhyperbolic. The following theorem classifies exceptional surgeries on wrapped Montesinos knots.

Theorem 5.5 Suppose $K=K^{a}\left(t_{1}, \ldots, t_{k}\right) \subset V$ is not equivalent to $K^{a}(0)$ or $K^{a}(1 / q)$ for any integer $q$. Let $(V, K, r)$ be an exceptional surgery. Then $(K, r)$ is equivalent to one of the following pairs. The surgery is small Seifert fibered for $r=1,2,3$ in (1) and $r=7$ in (4), and toroidal otherwise.

(1) $K=K^{0}$ (2) (the Whitehead knot), $r=0,1,2,3,4$.

(2) $K=K^{a}(n), n>2, r=0$ if $a=0$, and $r=2 n$ otherwise.

(3) $K=K^{a}\left(1 / q_{1}, 1 / q_{2}\right),\left|q_{i}\right| \geq 2$, and $r$ is the pretzel slope.

(4) $K=K^{1}(-1 / 2,1 / 3), r=6,7,8$.

Proof First assume that $k=1$, so $T[t]$ is a rational tangle. In this case any $K^{1}\left(t^{\prime}\right)$ is equivalent to some $K^{0}(t)$. By the above, the reciprocal $1 / t$ has the property that $K^{0}(t)$ is equivalent to $K^{0}\left(t^{\prime}\right)$ if $1 / t^{\prime}=2 \pm(1 / t)$, and by assumption $1 / t \neq 0,1$. Hence up to equivalence we may assume that $0<1 / t<1$, ie $t=p / q>1$. Note that $K_{n}$ is the 2-bridge knot in $S^{3}$ associated to the rational number $r=1 /(2 n+q / p)$. Hence if $q \neq 1$ then for any $n>1, K_{n}$ is not equivalent to a 2 -bridge knot associated to any rational number of type $1 /\left(b_{1}+1 / b_{2}\right)$ with $b_{1}, b_{2} \in \mathbb{Z}$. It follows from [3, Theorem 1.1] that all nontrivial surgeries on such $K_{n}$ are hyperbolic. By Theorem 4.3 this implies that $K \subset V$ admits no exceptional surgery. For $q=1, t=p / q>1$ is an integer. If $t=2$ then $K$ is the Whitehead knot in $V$ and it is well-known that $K$ admits exactly five exceptional surgeries as listed in (1). The hyperbolicity of $(V, K, r)$ for $r \neq 0, \ldots, 4$ can also be proved using the argument in the proof of Lemma 5.4 and the classification of exceptional surgeries on 2-bridge knots given in [3]. If $t>2$ then $K$ is not the Whitehead knot, and the argument in the proof of Lemma 5.3 shows that $\partial_{h} X$ is incompressible, hence by Theorem 3.8 we see that $K$ admits no Seifert fibered surgery and at most one toroidal surgery. Note that $K$ has a spanning surface $F$ in $V$ which is a once punctured torus or Klein bottle. As in the proof of Lemma 5.3, 
surgery along the boundary slope of $F$ produces a toroidal manifold, so there is no other exceptional surgery. The toroidal slope is given in (2).

We now consider the case that $k>1$. We may assume that $q_{i} \geq 2$ for all $i$ as otherwise the Montesinos tangle is equivalent to one with fewer rational tangles. If $k \geq 3$ then $K_{n}$ is a Montesinos knot of length at least 4 for all $|n| \geq 2$. By the author [24], we have $K_{n}$ admits no exceptional surgery. Hence by Theorem 4.3 we see that $K(r)$ is hyperbolic for all nontrivial $r$, so there is no exceptional surgery.

We now assume that $K=K^{a}\left(p_{1} / q_{1}, p_{2} / q_{2}\right)$ with $q_{i} \geq 2$. Then we have that $K_{n}=K\left(p_{1} / q_{1}, p_{2} / q_{2}, 1 / 2 n\right)$ or $K\left(p_{1} / q_{1}, p_{2} / q_{2}, 1 /(2 n+1)\right)$. By Theorem 4.3, if $(V, K, r)$ is exceptional then either $K_{n}\left(r_{n}\right)$ is toroidal for all but at most three $n$, or it is either reducible or atoroidal and Seifert fibered for all $n$. If $K\left(p_{1} / q_{1}, p_{2} / q_{2}, 1 / q_{3}\right)$ admits a toroidal surgery and $\left|q_{3}\right|>9$ then $\left|p_{i}\right|=1$ and the surgery slope is the pretzel slope, by [26]. Hence if $K_{n}\left(r_{n}\right)$ is toroidal for almost all $n$ then $K=K^{a}\left(1 / q_{1}, 1 / q_{2}\right)$ and $r$ is the pretzel slope, so (3) holds.

We may now assume $K_{n}\left(r_{n}\right)$ is reducible or atoroidal and Seifert fibered for all $n$. As above, we have $K_{n}=M\left(p_{1} / q_{1}, p_{2} / q_{2}, 1 / 2 n\right)$ or $K_{n}=M\left(p_{1} / q_{1}, p_{2} / q_{2}, 1 /(2 n+1)\right)$, and by [24] $K_{n}\left(r_{n}\right)$ cannot be reducible; hence it must be an atoroidal small Seifert fibered manifold for any $n$. By the author [27, Theorems 7.2 and 7.3], one of the following must hold:

(i) $K_{n}$ is a $\left(q_{1}, q_{2}, q_{3}, d\right)$ pretzel knot or its mirror image, and either $d=0$, or all $q_{i}$ are positive and $d=-1$. Moreover, either some $\left|q_{i}\right|=2$ or $\left|q_{i}\right|=\left|q_{j}\right|=3$ for some $i \neq j$.

(ii) $K_{n}=K(\mp 1 / 2, \pm 2 / 5,1 /(2 n+1))$.

In (i) above, the case $d=-1$ cannot happen in our case since $K_{n}\left(r_{n}\right)$ is atoroidal Seifert fibered for both $n$ positive and negative, contradicting the condition that all $q_{i}$ are of the same sign (up to taking mirror image of $K_{n}$.) Therefore $K_{n}$ must be a genuine pretzel knot if (i) holds. It follows that the tangle must be equivalent to $T\left[1 / q_{1}, 1 / q_{2}\right]$ or $T[-1 / 2,2 / 5]$.

The result now follows from Lemmas 5.3 and 5.4.

\section{References}

[1] J Berge, The knots in $D^{2} \times S^{1}$ which have nontrivial Dehn surgeries that yield $D^{2} \times S^{1}$, Topology Appl. 38 (1991) 1-19 MR1093862

[2] S Boyer, X Zhang, Reducing Dehn filling and toroidal Dehn filling, Topology Appl. 68 (1996) 285-303 MR1377050 
[3] M Brittenham, Y-Q Wu, The classification of exceptional Dehn surgeries on 2-bridge knots, Comm. Anal. Geom. 9 (2001) 97-113 MR1807953

[4] M Culler, C M Gordon, J Luecke, P B Shalen, Dehn surgery on knots, Ann. of Math. 125 (1987) 237-300 MR881270

[5] R Fintushel, R J Stern, Constructing lens spaces by surgery on knots, Math. Z. 175 (1980) 33-51 MR595630

[6] D Gabai, Foliations and the topology of 3-manifolds, III, J. Differential Geom. 26 (1987) 479-536 MR910018

[7] D Gabai, Surgery on knots in solid tori, Topology 28 (1989) 1-6 MR991095

[8] D Gabai, 1-bridge braids in solid tori, Topology Appl. 37 (1990) 221-235 MR1082933

[9] C M Gordon, Boundary slopes of punctured tori in 3-manifolds, Trans. Amer. Math. Soc. 350 (1998) 1713-1790 MR1390037

[10] C M Gordon, J Luecke, Dehn surgeries on knots creating essential tori, I, Comm. Anal. Geom. 3 (1995) 597-644 MR1371211

[11] CM Gordon, J Luecke, Toroidal and boundary-reducing Dehn fillings, Topology Appl. 93 (1999) 77-90 MR1684214

[12] C M Gordon, Y-Q Wu, Toroidal and annular Dehn fillings, Proc. London Math. Soc. 78 (1999) 662-700 MR1674841

[13] CM Gordon, Y-Q Wu, Annular Dehn fillings, Comment. Math. Helv. 75 (2000) 430-456 MR1793797

[14] C M Gordon, Y-Q Wu, Toroidal Dehn fillings on hyperbolic 3-manifolds, Mem. Amer. Math. Soc. 194 (2008) vi+140 MR2419168

[15] W Jaco, Lectures on three-manifold topology, CBMS Regional Conference Series in Mathematics 43, American Mathematical Society (1980) MR565450

[16] W Jaco, P B Shalen, Seifert fibered spaces in 3-manifolds, from: "Geometric topology (Proc. Georgia Topology Conf.”, (J C Cantrell, editor), Academic Press, New York (1979) 91-99 MR537728

[17] J M Montesinos, Surgery on links and double branched covers of $S^{3}$, from: "Knots, groups, and 3-manifolds (Papers dedicated to the memory of R H Fox)", (L P Neuwirth, editor), Ann. of Math. Studies 84, Princeton Univ. Press (1975) 227-259 MR0380802

[18] D Rolfsen, Knots and links, Mathematics Lecture Series 7, Publish or Perish, Berkeley, CA (1976) MR0515288

[19] M Scharlemann, Producing reducible 3-manifolds by surgery on a knot, Topology 29 (1990) 481-500 MR1071370

[20] F Waldhausen, Eine Klasse von 3-dimensionalen Mannigfaltigkeiten, I, II, Invent. Math. 3 (1967), 308-333; ibid. 4 (1967) 87-117 MR0235576 
[21] Y-Q Wu, Seifert fibered surgery on Montesinos knots arXiv:1207.0154

[22] Y Q Wu, Incompressibility of surfaces in surgered 3-manifolds, Topology 31 (1992) 271-279 MR1167169

[23] Y-Q Wu, The classification of nonsimple algebraic tangles, Math. Ann. 304 (1996) 457-480 MR1375620

[24] Y-Q Wu, Dehn surgery on arborescent knots, J. Differential Geom. 43 (1996) 171-197 MR1424423

[25] Y-Q Wu, Dehn fillings producing reducible manifolds and toroidal manifolds, Topology 37 (1998) 95-108 MR1480879

[26] Y-Q Wu, The classification of toroidal Dehn surgeries on Montesinos knots, Comm. Anal. Geom. 19 (2011) 305-345 MR2835882

[27] Y-Q Wu, Persistently laminar branched surfaces, Comm. Anal. Geom. 20 (2012) 397-434 MR2928718

Department of Mathematics, The University of Iowa 14 MacLean Hall, Iowa City, IA 52242-1419, USA

wu@math. uiowa. edu

Received: 25 September $2011 \quad$ Revised: 24 July 2012 